\title{
Téoros
}

Revue de recherche en tourisme

\section{Le camp de la jeunesse aux Jeux olympiques}

De la naissance à la reconnaissance

\section{Éric Monnin et Cyril Polycarpe}

Volume 33, numéro 1, 2014

Méga-événements sportifs

URI : https://id.erudit.org/iderudit/1036716ar

DOI : https://doi.org/10.7202/1036716ar

Aller au sommaire du numéro

Éditeur(s)

Université du Québec à Montréal

ISSN

0712-8657 (imprimé)

1923-2705 (numérique)

Découvrir la revue

Citer cet article

Monnin, É. \& Polycarpe, C. (2014). Le camp de la jeunesse aux Jeux olympiques : de la naissance à la reconnaissance. Téoros, 33(1), 20-31. https://doi.org/10.7202/1036716ar
Résumé de l'article

L'analyse des camps de la jeunesse aux Jeux olympiques (JO) nous montre que les jeunes participants se rassemblent en proposant des activités spécifiques dans la connaissance et l'accessibilité de l'olympisme grâce à la culture de la ville accueillante. La spécificité de ces camps tient dans le fait qu'ils rassemblent les notions de lieu et de patrimoine culturel, tant pour l'histoire olympique que pour la ville hôte, conférant une tendance à la mondialisation des valeurs olympiques au service du méga-événement et du lieu organisateur. Le camp de la jeunesse devient un objet de transmission décliné en catégories diverses : géographie, culture, sport, etc. Il participe ainsi à la dynamique des territoires et de l'évènement olympique en tant qu'acteur du tourisme national par la mise en avant de son patrimoine et social, par la transmission de savoirs vernaculaires. 


\title{
Le camp de la jeunesse aux Jeux olympiques De la naissance à la reconnaissance
}

\author{
Éric MONNIN \\ Maître de Conférences \\ Laboratoire C3S, UPFR Sports, Université de Franche-Comté \\ eric.monnin@univ-fcomte.fr
}

\section{Cyril POLYCARPE}

Doctorant STAPS, Laboratoire C3S (EA 4660)

Enseignant-chercheur

UPFR Sports, Université de Franche-Comté

cyril.polycarpe@univ-fcomte.fr

\begin{abstract}
RÉSUMÉ: L'analyse des camps de la jeunesse aux Jeux olympiques (JO) nous montre que les jeunes participants se rassemblent en proposant des activités spécifiques dans la connaissance et l'accessibilité de l'olympisme grâce à la culture de la ville accueillante. La spécificité de ces camps tient dans le fait qu'ils rassemblent les notions de lieu et de patrimoine culturel, tant pour l'histoire olympique que pour la ville hôte, conférant une tendance à la mondialisation des valeurs olympiques au service du méga-événement et du lieu organisateur. Le camp de la jeunesse devient un objet de transmission décliné en catégories diverses : géographie, culture, sport, etc. II participe ainsi à la dynamique des territoires et de l'évènement olympique en tant qu'acteur du tourisme national par la mise en avant de son patrimoine et social, par la transmission de savoirs vernaculaires.
\end{abstract}

Mots-clés: camps de la jeunesse, olympisme, valeurs, territoire, tourisme.

Ce texte interroge la manière dont la jeunesse est mise en scène par le Comité international olympique (CIO) lors des Jeux d'été et d'hiver (Monnin, 2012 : 23-26). Ainsi, étudier comment se sont élaborés les camps de la jeunesse aux JO et plus particulièrement leurs conditions de réalisations et de reconnaissance, nous renseigne sur l'identité et les usages culturels qu'ils développent. Nous comprendrons par ce biais le processus historique et social de la construction de l'identité du camp de la jeunesse, mais ainsi que celle du lieu (réel ou symbolique) où se déroulent ses activités. Notre démarche consiste à rechercher la signification de cet objet et, par conséquent, le sens des discours produits autour du «méga-événement» que représentent les JO pour la jeunesse (Polycarpe, 2013 : 455-463). Nous essaierons de saisir la culture olympique qui est véhiculée dans ces camps et qui forme un enjeu promotionnel à la fois pour le Mouvement olympique et pour la ville olympique d'accueil. Ainsi, les camps de la jeunesse de 1912 à 2004 nous permettent de comprendre l'identité spécifique qu'ils dégagent ainsi que leurs relations avec un territoire géographique dynamique durant le déroulement des JO.

Du 6 juillet au 22 juillet 1912, 1500 jeunes issus du monde Boy-scout des pays scandinaves et d'Allemagne convergent vers Stockholm pour la première rencontre internationale de la jeunesse olympique, lors de la cinquième olympiade sous l'autorité du roi de Suède, Gustave V. Selon les organisateurs, ces jeunes partagent une "condition semblable» telle que la «solidarité, l'entraide et le respect», d'après la philosophie du fondateur du scoutisme Lord Robert Baden-Powell. Après une longue pause, l'expérience est renouvelée en 1936, lors des Jeux de Berlin. Ces deux rassemblements ont pour point commun d'associer la jeunesse à la manifestation olympique, projet pédagogique affirmé et défini en ces termes : «Projet pédagogique d'éducation des jeunes élites du monde baptisé plus tard par Pierre de Coubertin "idée olympique" [...] une structure organisationnelle transnationale s'incarnant, dès juillet 1894, dans le Comité International Olympique»(Clastres, 2002 : 327). C'est en 1952, lors des JO d'été d'Helsinki, que l'occasion de réunir la jeunesse du monde est renouvelée, puis en 1998 à Nagano pour les Jeux d'hiver. Ce rassemblement de la jeunesse olympique se fait peu à peu le symbole des $\mathrm{JO}$ pour associer le plus grand spectacle sportif mondial à l'émerveillement de cette jeunesse âgée de 14 à 17 ans. Seuls deux Jeux font figure d'exception à cette tradition : à Melbourne en 1956, où le camp est remplacé par un programme de jeunes bénévoles composé essentiellement de scouts, et à Los Angeles, en 1984, où il est question d'un programme sportif préolympique. 


\section{Cadre conceptuel}

Les camps de la jeunesse corroborent l'évolution de l'histoire olympique, passant d'un objectif purement éducatif à un objectif politique, au sens le plus noble du terme qui est de contribuer à la paix et à la compréhension entre les humains. Cette innovation contribue à la réalisation de l'idéal olympique, et par conséquent, doit être évaluée dans cette optique. La jeunesse présente dans ces camps, qu'elle soit nationale ou extérieure, découvre la ville accueillante à travers son patrimoine architectural et culturel. Nous pouvons identifier ce phénomène comme un nouveau critère d'identité olympique. Ce sentiment d'appartenance à une communauté dépasse le seul cadre sportif des JO, car la ville organisatrice promeut aussi une image touristique mise en scène par le plus grand spectacle sportif mondial. Ces grands événements sont de formidables opportunités pour promouvoir, par le marketing territorial, la richesse des territoires organisateurs (Hatem, 2007). Conscientes de ces enjeux, les équipes d'organisation des JO sont attentives aux propositions concrètes émises par les camps pour intensifier les retombées économiques de ces événements sur le territoire national et vers l'international.

Le camp de la jeunesse olympique représente pour les participants une expérience unique et commune, notamment sur le plan humain et culturel (soirées culturelles, débats sur le thème des JO et de l'olympisme, etc.). Ces multiples activités invitent la jeunesse du monde à l'apprentissage des coutumes, des rites et du mode de vie de chacun des participants. Ainsi, ces regroupements de la jeunesse olympique préparent les jeunes individus de 14 à 17 ans à une «influence de l'effort sur la formation du caractère et le développement de la personnalité" afin d' "être un citoyen actif dans la société»(Coubertin, 1996 : 45). Ces rassemblements permettent d'évoquer la possibilité d'une idée de bien commun et ainsi, de favoriser la prise de conscience que l'olympisme est un instrument d'équilibre. En cela, il permet «de mettre le sport au service du développement harmonieux de l'humanité en vue de promouvoir une société pacifique, soucieuse de préserver la dignité humaine»(CIO, 2011: 10). Le paysage des camps de la jeunesse est traversé par une thématique centrale qui agite les consciences et mobilise les énergies, et qui a pour but de renouveler les dynamiques olympiques. Cet intérêt fait figure de nouveauté rafraîchissante, comme s'il permettait la régénération de structures à bout de souffle (Jennings, 2000). Le rôle et les missions des camps olympiques semblent voués à promouvoir, éduquer et former une jeunesse internationale à la thématique olympique. Ce sujet ouvre un éventail de possibilités en fonction des ambitions, de la stratégie de positionnement et des moyens disponibles pour une image attractive des villes (Sobry, 1993 : 69-73). Les exemples sont nombreux et parfois très différents sur le sens réel que nous devons accorder à l'orientation des camps de la jeunesse. Les organisateurs de ces camps ont-ils soutenu cette orientation parce qu'ils y voyaient un processus à construire? Servent-ils à raviver l'image d'un olympisme moderne par une internationalisation de leurs activités?

\section{Méthodologie}

Par le biais de l'histoire culturelle, nous analyserons la création des camps de la jeunesse aux JO, leurs caractéristiques et leur impact sur l'olympisme. Puis, nous présenterons l'analyse de leur contenu afin de montrer la manière dont sont utilisées les valeurs olympiques sur un plan d'attractivité culturelle et d'image auprès de la jeunesse présente lors des JO. Il s'agit d'étudier la restructuration de l'encadrement de la jeunesse avec le passage du processus existant à une structure en quête de légitimation lors du déroulement des JO. Cette affirmation leur confère une place inédite comme acteur de l'extension de l'olympisme dans notre société contemporaine, par l'intermédiaire de programmes culturels et éducatifs, et une patiente maturation d'intérêts communs pour diffuser une image positive de la philosophie olympique et de la ville organisatrice. S'agissant de dégager une dynamique, l'approche chronologique a été retenue pour montrer la continuité et les ruptures d'un processus soumis à de nombreuses révisions entre 1912 et 2004.

La consultation de fonds d'archives écrits du CIO, de la Revue olympique et de rapports officiels des Comités d'organisation des Jeux olympiques (COJO) sur la période concernée, constitue le corpus principal de l'étude. Les rapports officiels des COJO de 1912 à 2004 donnent des éléments chiffrés sur l'évolution des camps de la jeunesse et sont les sources des travaux les plus riches par leurs comptes-rendus de réunions, budgets, orientations pédagogiques, programmes d'activité, journaux internes. Les archives du CIO apportent quant à elles un décryptage des pratiques mises en place, la mise en œuvre des méthodes pédagogiques et les attentes souhaitées. La revue du CIO, la Revue olympique, permet de repérer des éléments déterminants au contexte socioculturel olympique. Les données extraites nous permettent en outre de dégager des éléments clés, à partir desquels nous affinons notre regard, d'une part sur l'identité spécifique qui caractérise le camp de la jeunesse, et d'autre part sur les caractéristiques mises en avant par le territoire d'accueil de l'événement olympique. L'analyse du contenu des actions menées durant ces camps se base sur différentes variables : leur positionnement philosophique et politique, leur statut, la définition de la «culture olympique» et le rôle qu'ils souhaitent jouer dans le Mouvement olympique. Il s'agit d'établir une histoire sérielle des mentalités (Rioux et Sirinelli, 1997) des camps de la jeunesse comme acteurs de mobilisation, d'intégration et de transformation des valeurs de l'olympisme.

\section{Résultats}

La genèse des camps de la jeunesse aux Jeux olympiques et leurs conséquences sur l'olympisme

Chaque camp de la jeunesse se construit en dehors de toute appartenance à la ville organisatrice afin de diffuser sa propre identité. Il apparaît sous différentes appellations : camp universel de la jeunesse (aux JO de Tokyo, en 1964), camp olympique mexicain de la jeunesse mondiale (en 1968, à Mexico), camp international de la jeunesse (en 1976, à Montréal) ou encore le camp olympique de la jeunesse (à Sydney, en 2000) (tableau 1). Fonctionnant comme un appendice de communication, le premier camp est organisé aux JO de Stockholm 


\begin{tabular}{|c|c|c|c|c|c|c|}
\hline \multicolumn{7}{|c|}{ Tableau 1 : Les camps olympiques de la jeunesse de 1912 à 2004} \\
\hline Jeux olympiques & $\begin{array}{l}\text { Nombre de } \\
\text { participants }\end{array}$ & $\begin{array}{l}\text { Nombre de } \\
\text { CNO }\end{array}$ & $\begin{array}{c}\text { Âge des } \\
\text { participants }\end{array}$ & Appellation du camp & Dates & Particularités \\
\hline Stockholm 1912 & 1200 & 4 & 12 à 18 ans & Camp olympique & $15 / 06$ au $15 / 07$ & $\begin{array}{l}\text { - uniquement des scouts } \\
\text { - sous tente }\end{array}$ \\
\hline Anvers 1920 & \multicolumn{6}{|c|}{ Pas de camp de la jeunesse } \\
\hline Paris 1924 & \multicolumn{6}{|c|}{ L'organisation des Jeux de l'enfance, sur trois jours, remplace le camp de la jeunesse } \\
\hline Amsterdam 1928 & \multicolumn{6}{|c|}{ Pas de camp de la jeunesse } \\
\hline Los Angeles 1932 & \multicolumn{6}{|c|}{ Pas de camp de la jeunesse } \\
\hline Berlin 1936 & 1700 & 23 & 15 à 18 ans & $\begin{array}{l}\text { Camp international } \\
\text { de la jeunesse }\end{array}$ & $27 / 07$ au $18 / 08$ & $\begin{array}{l}\text { - uniquement des garçons } \\
\text { - sous tente }\end{array}$ \\
\hline Helsinki 1952 & 2191 & 17 & 16 à 22 ans & & $10 / 07$ au $10 / 08$ & - sous tente \\
\hline Melbourne 1956 & \multicolumn{6}{|c|}{ Suppression du camp au profit d'un programme de jeunes bénévoles composés essentiellement de scouts } \\
\hline Rome 1960 & 1250 & 5 & 14 à 18 ans & $\begin{array}{l}\text { Camp international } \\
\text { de la jeunesse }\end{array}$ & $25 / 08$ au $12 / 09$ & - sous tente \\
\hline Tokyo 1964 & 1018 & 28 & 15 à 25 ans & Camp universel de la jeunesse & $6 / 10$ au $25 / 10$ & \\
\hline Mexico 1968 & 865 & 20 & 15 à 18 ans & $\begin{array}{l}\text { Camp olympique mexicain } \\
\text { de la jeunesse mondiale }\end{array}$ & $6 / 10$ au $30 / 10$ & \\
\hline Munich 1972 & 1640 & 53 & 17 à 20 ans & $\begin{array}{l}\text { Camp international } \\
\text { de la jeunesse }\end{array}$ & & $\begin{array}{l}\text { Le CIO reconnait le camp } \\
\text { comme un événement officiel }\end{array}$ \\
\hline Montréal 1976 & 925 & 45 & 17 à 20 ans & $\begin{array}{l}\text { Camp international } \\
\text { de la jeunesse }\end{array}$ & $13 / 07$ au $04 / 08$ & \\
\hline Moscou 1980 & 1085 & 54 & 17 à 20 ans & Camp international de jeunes & $17 / 07$ au $02 / 08$ & $\begin{array}{l}\text { Du 28/07 au 09/08 un autre camp } \\
\text { est organisé sur le site d'Olympie } \\
\text { en Grèce }\end{array}$ \\
\hline Los Angeles 1984 & \multicolumn{6}{|c|}{ Suppression du camp au profit d'un programme sportif préolympique } \\
\hline Séoul 1988 & 882 & 43 & 18 à 22 ans & Camp international de jeunes & $13 / 09$ au $02 / 10$ & \\
\hline Barcelone 1992 & 497 & 67 & 18 à 22 ans & Camp international des jeunes & $23 / 07$ au $10 / 08$ & \\
\hline Atlanta 1996 & 458 & 152 & 16 à 18 ans & Camp olympique de la jeunesse & $17 / 07$ au $31 / 07$ & \\
\hline Nagano 1998 & 217 & 51 & 15 à 20 ans & Camp des Snowlets & $04 / 02$ au $19 / 02$ & Premier camp aux Jeux d'hiver \\
\hline Sydney 2000 & 381 & 170 & 16 à 18 ans & Camp de jeunesse olympique & $12 / 09$ au $04 / 10$ & \\
\hline Athènes 2004 & 450 & 200 & 16 à 18 ans & Camp olympique de la jeunesse & $11 / 08$ au $26 / 08$ & \\
\hline
\end{tabular}

en 1912, et souhaite avant tout diffuser une image dynamique de la jeunesse suédoise. Le CIO et Pierre de Coubertin ne s'y opposent pas, pour preuve : «le Comité International Olympique donnait une conclusion inattendue à la $\mathrm{V}^{\mathrm{e}} \mathrm{olym}-$ piade en décernant la médaille olympique à Madame Wersäll née comtesse Lewenhaupt, dont six fils ont pris part aux Jeux de 1912, les derniers en qualité de boy-scouts» (Coubertin, 1996 : 126). Afin de s'assurer le plus grand succès, les scouts suédois lancent des invitations aux autres scouts allemands, anglais, danois, français et norvégiens. Seuls les Allemands, les Danois et les Norvégiens répondent positivement. Cette présence scoute aux Jeux permet de mettre en avant leur savoir-faire en matière d'organisation et de légitimer leur existence et leur présence grâce à leur vécu, leurs connaissances et leurs compétences dans un territoire olympique en quête d'image positive.

Chaque jour, quelque 600 scouts (Bergvall, 1912 : 812-817) se relaient pour assurer le bon fonctionnement des épreuves, notamment au stade olympique. À titre de remerciement, le comité d'organisation les autorise, le 12 juillet, à présenter leurs manifestations dans le stade olympique. Plus de mille scouts suédois ont effectué des démonstrations de gymnastique et 
quelques mouvements de respiration. Puis, les Danois ont pris le relais pour présenter des jeux typiques de leur pays. Cette présentation devant le public du stade olympique a été appréciée et fortement applaudie. En laissant s'exprimer les scouts scandinaves dans le stade olympique, le CIO témoigne toute sa reconnaissance à ce mouvement de la jeunesse. Pour la Suède, il s'agit d'afficher la vitalité de sa nation et de sa jeunesse par un processus de reconnaissance de leurs spécificités sportives à l'international. C'est dans cette articulation entre l'identité exprimée par les individus, en l'occurrence ici les scouts, et l'identité pour autrui que naît une reconnaissance auprès du $\mathrm{CIO}$ pour cette manifestation de la jeunesse. Autrement dit, le contexte national et international interagit pour définir cette association avec l'olympisme. Par conséquent, dès 1912, le camp olympique de la jeunesse a profité du soutien politique de la monarchie suédoise et du prétexte de l'expansion du scoutisme, qui structure la jeunesse de la société scandinave depuis 1910, pour accompagner une première application des principes de l'éducation olympique définie par Pierre de Coubertin (Monnin, 2012 : 333-351).

Avant que n'intervienne la Seconde Guerre mondiale, seuls les Allemands, à l'occasion des Jeux de Berlin en 1936, renouent avec l'organisation d'un camp olympique de la jeunesse qui se tourne vers les étudiants, les adolescents et les enfants de l'organisation paramilitaire Hitlerjugend (jeunesse hitlérienne). Ce rassemblement regroupe environ « 1700 jeunes de 15 à 18 ans, choisis parmi l'élite des écoles d'Éducation et physique dans 23 pays, qui se réunissent de nouveau sous la tente, au pied du stade» (Richeter et OCOG Berlin, 1937 : 1138-1147). La pratique de ce camp de la jeunesse se concentre sur des manifestations culturelles et folkloriques, des excursions et une participation au spectacle du Festival olympique "Olympic Youth», dans le stade olympique de Berlin. Ce fort volontarisme politique préside à la valorisation de l'intérêt des camps de la jeunesse lors des JO. La découverte de la culture nationale du pays d'accueil et la connaissance de l'autre sont parmi les enjeux de cette manifestation culturelle.

Dans les faits, il apparaît que l'organisme est en réalité étroitement subordonné. Après ces deux premières expériences à Stockholm et à Berlin, les COJO sont plus au service de la promotion et de la propagande de leurs politiques sportives et nationales que de l'olympisme même. Le caractère puissant de la nation y est exacerbé à travers sa jeunesse qui devient la vitrine du dynamisme patriotique, où la promotion de l'éducation physique et sportive sert de base à la «régénérescence» nationale. Les démonstrations de ces deux camps de la jeunesse aux JO nourrissent le fantasme d'une politique volontariste d'éducation à la cause olympique (Matard-Bonucci et Milza 2004 : 151). Dans le contexte d'une crise de civilisation, les camps de la jeunesse sont l'expression de la vigueur culturelle et physique des entités nationales. L'esprit de ces regroupements devient le produit d'une histoire commune au passé antique affirmé, sous la forme d'un "mythe mobilisateur». L'histoire gréco-romaine et chrétienne de la civilisation s'identifie dans le message olympique, puis est déclinée dans la culture nationale à travers l'olympisme assimilé dans l'organisation des camps de la jeunesse : «Dans le cœur de chacun de nous, brille l'idée olympique, l'idée qui par de chevaleresques combats sportifs anoblira ou, dans une certaine mesure, spirituel sera les rapports entre les peuples. Car au sein de l'idée olympique s'unissent la pensée du sport qui réunit les peuples modernes et la tradition de la culture antique, piliers fondamentaux de notre civilisation» (Lippert, 1936:8).

Ce projet culturel manque de repères clairs, notamment en matière de politique éducative de la part du CIO et des COJO, car l'effort doit être concentré auprès d'une jeunesse qui constitue l'avenir olympique. Ce renforcement se doit de combattre les excès existants entre la force et la suprématie, la grandeur d'un côté et les effets de démonstration de l'autre. Il est donc nécessaire de permettre à l'olympisme de s'enraciner dans l'esprit des athlètes et des dirigeants sportifs par une valorisation de la culture et des échanges. Ce processus nécessite de la part des dirigeants olympiques la définition d'un concept d'olympisme commun et essentiel à la création d'un sentiment d'appartenance fort et démonstratif. L'accent mis sur une " unité de culture», reconnue comme un héritage culturel fondateur, laisse toute une série d'ambiguïtés empreinte de nationalisme comme base d'une conscience commune. Selon Carl Diem, il existe une histoire commune, un patrimoine de "vingt-cinq siècles» qui établit un "esprit olympique» et une «société» de peuples (Diem, 1950 : 22-23). Cependant, cette conscience olympique commune est appropriée par la nation, à défaut d'une échelle olympique aux règles définies par la Charte olympique et le CIO.

Dans ce contexte, les cultures scandinaves et allemandes présentent une forme de syncrétisme culturel avec la culture olympique. Les cultures nationales, par l'organisation des JO, veulent «s'olympiser» pour une reconnaissance internationale. La sortie de guerre en 1945 est confrontée à une autre approche de l'olympisme, qui met en avant la nécessité d'une coopération culturelle entre les Comités nationaux olympiques (CNO) participants, afin de consolider une «culture olympique» menacée. En 1946, le président du CIO Johannes Sigfrid Edström déclare à l'occasion de la première Session de Lausanne en 1946 que «la flamme olympique est toujours vivante. Bientôt elle brûlera dans tout son éclat et éclairera le monde» (Edström, 1946 : 10). Cet idéalisme d'ouverture à la coopération culturelle et organisationnelle trouve un premier aboutissement dans la création de la Journée olympique mondiale lors de la Session olympique du 29 janvier 1948 (Procès-verbal du CIO, 1948), mais l'austérité et le manque de dynamisme pédagogique de cette initiative n'ont pas l'écho escompté.

L'intérêt du camp olympique est relancé en 1952, avec un "esprit olympique» renouvelé par une politique éducative centrée sur l'olympisme et la diffusion de la culture nationale. La mobilisation de 2191 participants au camp des JO d'Helsinki pour suivre un programme culturel et sportif relance cet objet de communication pour l'olympisme et l'image du territoire dans lequel il s'inscrit (illustration 1). À Rome, en 1960, 1250 jeunes gens, hébergés sous tentes, participent à des activités similaires (visites de villes, centres historiques, etc.) et sont invités au Vatican par Jean XXIII pour une audience papale. 


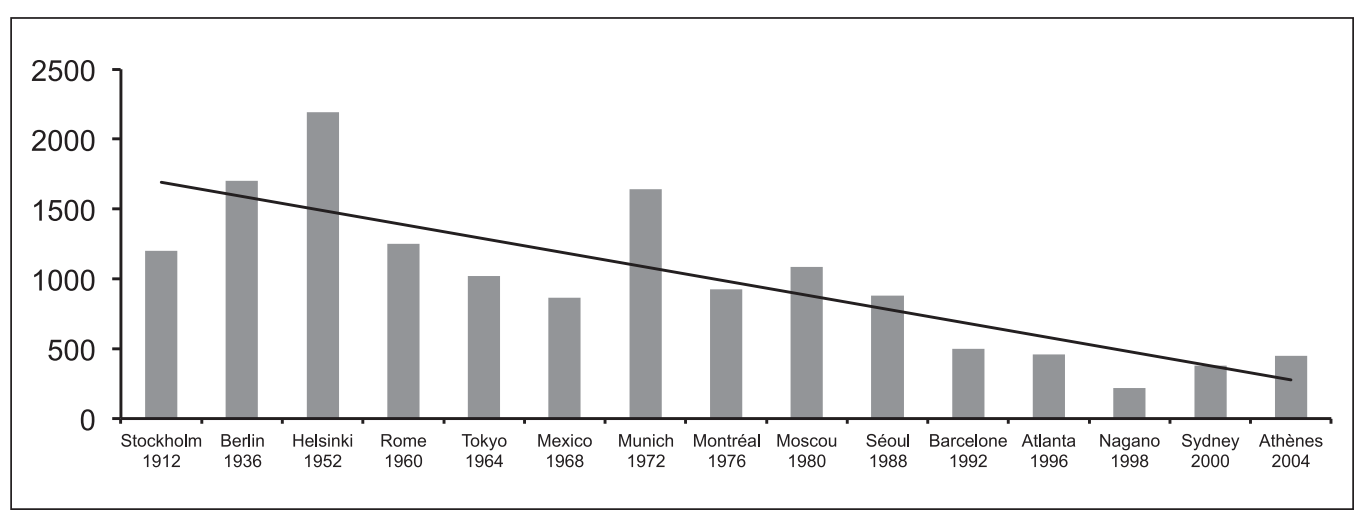

ILLUSTRATION 1 : Nombre de participants au camp de la jeunesse depuis 1912 (source : compilation des auteurs).
En 1961, la création de la commission pour l'Académie internationale olympique (AIO) permet le développement d'une politique éducative dans un cadre olympique chez les jeunes de 14 à 17 ans. L'établissement de relations, d'échanges culturels et artistiques entre les différents jeunes des nations présentes à Helsinki en 1952 facilite la compréhension du sens du message olympique et de ses valeurs. Dans le cas particulier des Jeux d'Helsinki en 1952, la question du rapprochement culturel par la jeunesse internationale prend un caractère plus exacerbé et symbolique dans un contexte international lourd et pesant. Par conséquent, les initiatives entreprises en faveur d'une ouverture entre les peuples sont encouragées. Au-delà des particularités idéologiques entre les nations présentes, se dégage une identité spécifique sur une base de collaboration et d'échanges entre les jeunes venus du monde entier.

À partir de cette olympiade, cette manifestation de la jeunesse fait véritablement partie du paysage olympique car elle offre une sélection de valeurs qui expriment un point de vue singulier sur un environnement et sur les éléments qui le composent. Les camps de la jeunesse d'Helsinki, de Rome et de Tokyo nous renseignent sur le rapport de la ville d'accueil à une manifestation et à un espace qui est à la fois géographique, culturel et social. L'objectif est de transformer le jeune participant en un olympien accompli en utilisant sa vision du monde tout en l'impliquant. Ainsi, les JO de Tokyo (1964), de Mexico (1968) et de Munich (1972) développent la satisfaction d'un plaisir de participation et d'une estime de soi de chaque participant suffisamment forte pour entreprendre leur reconnaissance auprès des instances olympiques.

À l'occasion des Jeux de Mexico où sont rassemblés 865 participants et 20 CNO (Comités nationaux olympiques), pour la première fois, un comité d'organisation en lien direct avec le COJO mexicain est mis en place : «le Comité organisateur des Jeux de la XIX ${ }^{e}$ olympiade a obtenu, pour ce programme, le patronage de l'Institut National de la jeunesse mexicaine» (CIO, 1968 : 17). À l'issue de ces Jeux, les camps de la jeunesse développent une image positive pour la ville d'accueil à l'international. La représentation des images véhiculées est autant valorisée que le contenu. Ainsi : «les chefs de délégation demandent au CIO d'inclure désormais le camp de jeunesse dans le programme olympique. Le Comité ne répond pas à cette requête, mais conserve son attitude de tolérance envers le camp » (CIO, $1968: 17)$.
Depuis cet événement, le CIO mentionne et recommande dans la Charte olympique l'organisation d'un camp de la jeunesse durant chaque Olympiade. Sur cette base, le COJO des Jeux de 1972 décide de bousculer les habitudes et d'intégrer le camp aux JO. Le CIO est contraint d'accepter cette initiative et reconnaît l'existence du "camp de la jeunesse comme un événement officiel» (COJO Munich, 1974).

Soixante ans ont donc été nécessaires pour que le camp de la jeunesse puisse compter dans l'organisation des Olympiades, à l'exception des JO de Los Angeles de 1984. Le comité d'organisation préfère mettre en place un programme sportif préolympique pour la jeunesse. La remise en cause du camp de la jeunesse par les autorités américaines provoque une forte réaction, et des jeunes de pays d'Europe, en particulier de la République fédérale d'Allemagne, décident de l'organiser «officieusement et dans le désir de sauvegarder la tradition du camp» (SLOOC, 1989 : 465). Ce rassemblement permet un processus de conscientisation révélant une nouvelle vision pour l'olympisme et pour l'image des JO dans la ville d'accueil. Cette "conscience olympique» n'a pas été clairement identifiée par les dirigeants du COJO de Los Angeles, qui n'ont mesuré ni le sens ni la portée du message de cette jeunesse dans la mise en scène du méga-événement que constituent les Jeux.

De 1912 à 2004, quinze camps ont été organisés, dont un durant les Jeux d'hiver de Nagano en 1998 (tableau 2). Le camp se destine à la promotion des échanges entre les jeunes, avec pour objectif de resserrer les liens entre les nations et de renforcer la compréhension mutuelle dans la construction d'un territoire où les distances s'effacent par la proximité des valeurs olympiques partagées. L'objectif essentiel est d'assurer durablement l'amitié entre les nations à travers des rencontres et des conférences qui mélangent sport, culture et éducation (Charte olympique, 2013 : 11). À l'issue du camp de Nagano, la participante japonaise Mio Sasaki déclare : «Je crois que j’ai enfin compris la véritable signification de l'expression échange international» (Revue olympique, $1998: 30$ ).

\section{Promotion d'un espace d'échanges interculturels}

Le volet éducatif des camps de la jeunesse connaît une dynamique renouvelée depuis la fin de la Seconde Guerre, car le choc entraîne une mutation de la perception du rôle de la jeunesse dans le Mouvement olympique. Cette influence est 
Tableau 2: Le camp des Snowlets aux Jeux olympiques d'hiver de Nagano

\begin{tabular}{|c|c|c|c|c|c|c|c|c|}
\hline $\begin{array}{l}\text { Jeux } \\
\text { Olympiques }\end{array}$ & $\begin{array}{l}\text { Nombre de } \\
\text { participants }\end{array}$ & $\begin{array}{l}\text { Nombre de } \\
\text { CNO }\end{array}$ & $\begin{array}{c}\text { Âge des } \\
\text { participants }\end{array}$ & $\begin{array}{l}\text { Appellation du } \\
\text { camp }\end{array}$ & Dates & Particularités & Lieux & $\begin{array}{l}\text { Thèmes } \\
\text { du camp }\end{array}$ \\
\hline Nagano 1998 & 217 & 51 & 15 à 20 ans & $\begin{array}{l}\text { Camp des } \\
\text { Snowlets }\end{array}$ & $04 / 02$ au $19 / 02$ & $\begin{array}{l}\text { Premier camp } \\
\text { aux Jeux d'hiver }\end{array}$ & $\begin{array}{l}\text { Maison des } \\
\text { séminaires de } \\
\text { l'université Nihon } \\
\text { à Kuruizawa }\end{array}$ & $\begin{array}{l}\text { Un monde solid- } \\
\text { aire; partageons } \\
\text { l'amour; parta- } \\
\text { geons la paix }\end{array}$ \\
\hline
\end{tabular}

Source : compilation des auteurs.

une réaction aux excès antérieurs qui ont rendu nécessaire un changement de nature des initiatives à destination de cette jeunesse. Le président du CIO exprime cette volonté en ces termes : «Le monde aspire à la paix. L'avenir appartient à la jeunesse. Pour celle-ci, l'idée olympique ne signifie pas seulement une coopération internationale pour l'amélioration physique si nécessaire après ce terrible conflit mondial, mais aussi un désir ardent de collaboration pour le retour à la paix » (Edström, 1947 : 1). Cette forme d'éducation crée les conditions par lesquelles l'idéologie olympique peut devenir une expérience unique en son genre, capable d'offrir des modes de conduite et d'action dans une situation donnée à chaque personne ou à un groupe. Le camp de la jeunesse aux JO n'est pas seulement une élaboration festive et culturelle, il est une construction sociale.

Ainsi, les COJO s'appuient sur les camps pour promouvoir un espace «d'échanges culturels» (COOB' 92, 1992 : 359) sous la direction du Mouvement olympique. Dans cet espace, de multiples activités sont organisées suivant la configuration d'un territoire et les usages qui y sont développés pour servir la revendication identitaire olympique et locale : visite des épreuves olympiques, cérémonies olympiques, manifestations culturelles et folkloriques, ateliers de discussion, etc. Les organisateurs valorisent l'héritage culturel national. Aux Jeux de Tokyo, en 1964, le camp installé dans les bâtiments de l'université Gakugei permet aux 1018 participants de prendre part aux différentes représentations folkloriques de chaque nation présente, de visiter des établissements industriels et culturels de la capitale nipponne tout en participant à certaines épreuves olympiques. Quant aux Jeux de Mexico, en 1968, l'essentiel est de promouvoir l'interculturalité entre les participants. Mme Diana Salvat et M. Alberto Campillo Saenz, responsables du camp mexicain, ont installé celui-ci au centre récréatif Oaxtepec. Cet endroit mythique a été le lieu de villégiature préféré des empereurs aztèques. Dans cet espace chargé d'histoire et propice aux échanges, les participants "ont d'emblée fraternisé avec leurs compagnons en provenance des quatre points cardinaux. Ils ont organisé des jeux, chanté, dansé, échangé des souvenirs et des objets typiques, confronté leurs langues, leurs expériences, leurs coutumes et leurs idées» (OCOG Mexico, 1969).

Le camp a favorisé l'instauration d'un lieu de conscience à partir d'un lieu de mémoire, qui devient le symbole d'une relation entre passé et présent dont le centre est la jeunesse et le partage. Cette initiative démontre que le lieu d'implantation du camp favorise l'échange culturel et le rapprochement des peuples. Elle ouvre aussi un nouveau volet dans le dossier de candidature d'une ville olympique : celui de la mise en avant de son patrimoine au service des nouvelles générations pour sa (re)découverte à l'occasion d'échéances internationales (Gold et Gold, 2007). Le camp olympique s'immerge et s'adapte au milieu dans lequel il est organisé pour devenir un support de «services et de guide patrimonial» : services cognitifs, services sportifs et richesse culturelle locale. Les camps de jeunesse deviennent un capital symbolique pour le Mouvement olympique, pour la valorisation de ses valeurs, et pour le COJO comme déclinaison symbolique. Cette politique culturelle et éducative intense a pour objectif de définir plus précisément des valeurs et des principes jusque-là flous tant sur la connaissance de l'olympisme que sur le patrimoine historique de la ville olympique. Comme le souligne la Charte olympique, le camp est un outil de communication des fondements de l'olympisme dans un but de paix entre les peuples et les individus. Par exemple, durant les Jeux de 1972, à Munich, en plus des visites culturelles (théâtre, musée, folklore, visites d'usines et d'instituts scientifiques), sont organisées des campagnes d'information permettant de sensibiliser la jeunesse au passé de la ville olympique. Le COJO de Munich n'hésite pas à faire table rase de son passé en montrant les rapports étroits que leur ville et le nazisme ont entretenus tout en rappelant les actions allemandes d'opposition au régime. Le 25 août 1972, 600 participants se sont rendus au camp de Dachau où une cérémonie du souvenir et de recueillement a été organisée.

La surabondance des signes positifs sur la présence de cette jeunesse internationale aux Jeux et à la ville olympique entraîne une structuration interne et indépendante à Montréal, en 1976, en éditant quotidiennement un journal. Douze de ses membres rédigent un quotidien intitulé «Bonjour», dans lequel apparaissent les nouvelles du camp et les activités menées par les différents groupes. Ce journal permet d'instaurer un lien entre chacun et de faciliter les échanges entre tous : "communications et fraternisations sont les mots d'ordre du camp» (OCOG Montréal, 1978). Le symbole du camp est décrit à l'ensemble des jeunes présents comme un rappel afin de réactiver la mémoire de chacun sur les connaissances et les repères de l'olympisme : «l'emblème du camp international de la jeunesse de 1976 est en forme de fleurs. Les cinq pétales symbolisant les cinq continents se déploient autour du réceptacle floral représenté par l'emblème des jeux de la XXI ${ }^{e}$ olympiade» (Bonjour, 1976 : 4). Ce rayonnement symbolique se nourrit de l'expérience olympique, avec la création d'un emblème auquel sont ajoutés un thème principal 
et un nom lors de chaque camp des olympiades suivantes. Pour les Jeux de Nagano en 1998, le «Camp des Snowlets» (Monnin, 2013 : 145-153) a pour thème principal « un monde solidaire». Ces différentes initiatives font émerger l'«esprit olympique» et promeuvent l'olympisme au sein des camps de la jeunesse. L'espace et sa culture sont les supports d'une identité olympique qui s'en nourrit. Pour faciliter les échanges, le camp doit comporter de multiples installations. À Moscou en 1980, «des salles de lecture et de musique, des ateliers, des installations sportives, des cours d'artisanat et de civilisation mexicaine, des conférences, des festivals folkloriques avec le défilé de costumes régionaux, des soirées dansantes, des veillées et des sérénades" (Novikov et OCOG Moscou, 1981 : 42). Les moyens utilisés favorisent les échanges, les discussions et permettent d'établir des liens nouveaux avec l'ensemble des participants. Cependant, une salve de critiques, notamment dans le journal Le Monde, dénonce le contenu politique de ce rassemblement qui affiche plus l'ambition de démontrer la richesse du communisme que de promouvoir l'olympisme (Le Monde, 1980).

La connaissance de l'olympisme devient un enjeu et une évidence au sein de la plupart des camps de jeunesse : celle de transmettre les émotions des épreuves olympiques et de favoriser les échanges interculturels. À l'issue du camp de la jeunesse d'hiver de Nagano, le 17 février 1998, un appel est lancé au reste du monde dans un but d'amitié pour promouvoir l'olympisme comme symbole de paix universelle. Cette «Déclaration du camp international de jeunesse de Nagano» est une réflexion remplie d'intention et menée sur une logique de participation afin d'obtenir un avantage durable, celui de s'octroyer un soutien indéfectible d'une jeunesse qui représente l'avenir de l'olympisme. Cette segmentation du public jeune répond à une stratégie d'intéressement pour s'imposer dans le développement des valeurs olympiques et pour en construire une culture connue et reconnue de tous.

Soutenues vigoureusement par le CIO et les COJO, les actions de promotion de l'olympisme font de la coopération entre les peuples une priorité pour insuffler une vraie impulsion internationale qui sert aussi les intérêts de la ville olympique. Ces initiatives bénéficient d'un écho important dans la mesure où elles répondent clairement à une attente formalisée depuis 1952 par les camps de la jeunesse. Pour autant, au vu de la rapidité avec laquelle ceux-ci se déploient et de l'engouement qu'ils suscitent, il semble peu probable qu'ils émergent ex nihilo au début des années 1950. L'étude attentive de ces camps depuis 1912 incite à penser que la préoccupation de la représentation de l'olympisme est déjà un projet important (ou tout du moins est-il en débat) dans les COJO. Cette observation nous amène à formuler l'hypothèse selon laquelle la création des camps de la jeunesse ne fait en réalité qu'entériner un processus déjà bien engagé. En d'autres termes, l'impact d'une dynamique olympique sur les plans culturel et géographique grâce à la jeunesse est perçu par les dirigeants des JO dès 1912. Cette voie s'inscrit dans un projet de promotion symbolique de l'olympisme à partir de valeurs du temps présent. La mise en place de rituels, de débats, de démonstrations sportives et culturelles font de la jeunesse un enjeu pour le présent et l'avenir aux yeux des dirigeants olympiques.
La modernisation et l'adaptation du message olympique aux nouvelles attentes des jeunes, notamment en termes d'internationalisation des échanges et des actes, permettent d'insérer dans l'olympisme un rapport culturel propre à chaque COJO, comme lors des Jeux de Stockholm (1912). Afin d'obtenir une légitimité internationale, le comité d'organisation suédois expose et présente au reste du monde sa culture sportive (exercices de gymnastique). L'exposition de cette culture sportive nationale par le lien olympique devient un enjeu primordial pour les villes hôtes des JO. Il importe, dans ce cadre, de se plonger dans l'histoire du positionnement des camps de la jeunesse pour en saisir la signification. Notons que choisir cette posture interprétative permet de mettre en perspective l'engouement pour l'olympisme, car les données éducatives et sportives sont de plus en plus employées à travers des groupements de jeunesse (Augustin et Ion, 1993). Toutefois, le statut contesté des JO dans la société (contestation économique, culturelle et environnementale) permet l'éclosion et le développement des camps de la jeunesse comme innovation régénératrice.

\section{Modernisation et internationalisation des camps de la jeunesse}

L'analyse des contenus des camps de la jeunesse fait apparaître une histoire mouvementée qui se révèle structurante afin de dévoiler les forces qui modèlent les identités de ces camps. Dans le contexte de 1912, les camps de la jeunesse ont nécessairement à se positionner sur la construction de l'olympisme, et plus particulièrement sur la question de fraternité entre les peuples comme symbole d'identité. Ce sont autant de valeurs à défendre, à protéger et à construire qui permettent de désigner les pratiques, les représentations et les attitudes de ces camps dans l'expansion de l'olympisme sur le plan international. Or, cet engagement dans la coopération avec leurs homologues présents est loin d'être évident, sauf dans le cadre régional et sur la base du scoutisme à Stockholm. En 1936, le contexte est pesant face à la suspicion et à un hermétisme total. Les positions des dirigeants organisateurs sont totalement orientées vers la démonstration de force (Brohm, 2008 : 47). L'ampleur de l'enjeu de l'organisation des Jeux de 1936, revenant en Europe après la réussite américaine des Jeux de Los Angeles en 1932, dépasse la discorde du boycottage de Berlin et de la contre-manifestation de Barcelone (Clastres, 2008 : 71-73). Dans cette optique, une vaste politique culturelle et socio-éducative se développe dans les camps de la jeunesse de Berlin en bénéficiant d'un budget important et d'une grande autonomie face au CIO. Le service d'éducation du III ${ }^{\mathrm{e}}$ Reich, que dirige Hans von Tschammer und Osten, met en place des expériences novatrices en finançant des plaquettes, en encourageant la formation de cadres et en réactivant le réseau des grandes manifestations de masse telle que celle des Hitlerjugend. Dans ce cadre, l'action du pouvoir nazi, en particulier la jeunesse hitlérienne et la jeunesse étudiante nazie, s'implante solidement dans une démonstration disciplinaire et a pour but de montrer les réformes éducatives nazies au moment de la reconnaissance internationale du régime hitlérien hors de l'olympisme. 
Les camps de la jeunesse s'intègrent initialement très peu dans ces desseins culturels éducatifs nazis. Pour bon nombre de responsables qui condamnent sans appel le spectacle allemand, il n'est pas question de se rendre de l'autre côté du Rhin et encore moins de s'ouvrir à la jeunesse allemande. Des manifestations se déroulent en Europe et aux États-Unis d'Amérique contre la tenue des Jeux de Berlin; en France, la Fédération sportive et gymnique du travail (FSGT) lance le slogan : "Pas un sou, pas un homme pour les JO de Berlin!» (FSGT, 1936). Si le sens commun, issu de la philosophie de l'olympisme, tend à faire croire que les camps exigent naturellement un esprit de coopération internationale de leur part, à l'inverse, les organisations allemandes le vivent comme un «redressement» éducatif national et excluent tout échange avec le communisme dans ces mouvements de jeunes.

Une inflexion dans le rôle des camps olympiques est palpable à partir de 1948. En effet, au lendemain de la Seconde Guerre mondiale et de la Session du CIO à Lausanne en 1946, la politique olympique internationale se trouve modifiée par un rapprochement, puis par la reconnaissance du CNO de l'URSS à la Session olympique de Vienne (6-7 mai 1951) (Peppard et Riordan, 1993 : 62). Désormais, les camps de la jeunesse s'ouvrent très progressivement au contexte international. Quelques faits saillants montrent qu'un changement de mentalité vis-à-vis des relations au sein du CIO est, à terme, incontournable. La déclaration «d'amende honorable» (Bulletin du Comité International, 1950 : 21) est faite par l'Allemagne en 1950 en marge de la réunion de la Commission exécutive de Lausanne (du 25 au 29 août 1950), sur leurs responsabilités dans la guerre. Cet acte caractérise les formes de pratique de l'olympisme et les formes de territorialisation des camps d'après-guerre à une échelle internationale, à l'image de ceux de Rome ou de Tokyo.

Depuis 1968, il est également à noter que les responsables des camps de la jeunesse choisissent le lieu des regroupements pour leurs manifestations. Ce choix se justifie symboliquement, souvent par l'affirmation de la nécessité d'envisager l'avenir de ces regroupements avec la jeunesse internationale. L'accroissement de la sensibilité de la jeunesse à l'olympisme tient aussi à la proximité des Jeux qui sont un lieu de rencontre, un vrai carrefour d'idées et de «débat public». Depuis 1952, les conférences, les rencontres et les séminaires résonnent dans ces milieux culturels et socio-éducatifs concernés par l'olympisme. Les mobiles qui sous-tendent les rapports des camps comme des acteurs de socialisation au message olympique se révèlent être un concept de régénération qui consiste à retrouver une dynamique structurelle et internationale. Lorsque le camp olympique de la jeunesse, aux Jeux de 1972, est désigné comme partie intégrante du Mouvement olympique par le $\mathrm{CIO}$, un mythe olympique prend forme par la mise en place d'une éducation olympique spécifique. Les camps sont mis en action par la coopération qui y règne, malgré le poids des cultures nationales exposées et aménagées, ou d'un contexte international qui a largement entravé les échanges. En centrant l'intérêt des camps sur l'éducation olympique, se crée une dynamique d'engagement et de connaissance à l'échelle internationale. Initialement motivée par défaut, l'éducation olympique prend d'abord pour objectif de «surmonter le passé» : désormais les camps fondent une représentation positive des valeurs olympiques à chaque Jeux. Les images développent un argumentaire d'ordre fonctionnel en montrant les activités proposées mais également par les promesses symboliques et métaphoriques de chaque manifestation. Les camps de la jeunesse en cours de légitimation deviennent un facteur de connaissance des valeurs de l'olympisme et d'internationalisation. Ils constituent une réelle possibilité d'expansion et de réhabilitation en termes d'image pour le CIO avec une incorporation des éléments constitutifs de l'olympisme sous forme de projection et d'introjection mêlant échange, ouverture vers l'autre et pacifisme. Cette communion du sensible et du culturel apparaît étroitement liée à une quête spirituelle qui en fait une forme novatrice pour la ville olympique et le CIO.

Dans le cadre de sa résolution culturelle, le CIO considère que le camp olympique de la jeunesse doit reposer sur une "union culturelle», afin de promouvoir l'olympisme et la mémoire nationale du lieu d'organisation. Cependant il s'avère rapidement que les COJO ne sont pas disposés à remettre en cause leur indépendance culturelle, car ils veulent aussi promouvoir leur culture nationale. On ne parvient pas à s'entendre sur le concept de «collaboration culturelle collective » et à se mettre d'accord pour définir une action culturelle commune. Au cours des Jeux de Moscou en 1980, l'omniprésence de drapeaux du CIO et de la mascotte olympique ne créent pas une atmosphère chaleureuse (Gygax, 2002 : 487-509).

Par l'intermédiaire de la Charte olympique, les organisateurs proposent de développer une identité spécifique grâce à une coopération culturelle entre les jeunes des $\mathrm{CNO}$ reconnus. L'objectif premier est de sensibiliser les jeunes à l'olympisme sous la forme d'une culture commune, puis de développer une conception militante de l'olympisme afin de promouvoir un sentiment d'appartenance à cette culture. La création d'échanges est recommandée lors des JO afin de pérenniser les relations menées lors des précédents regroupements. Pourtant, ce caractère n'est pas ou peu suivi dans les faits. Les camps de la jeunesse servent donc de guide «spirituel et pratique» au $\mathrm{CIO}$ et à la ville olympique où le participant se projette dans un imaginaire décrit et espère bénéficier du symbolisme olympique auquel il est associé. Ainsi, le participant au camp a le sentiment d'être actif, de s'affranchir des barrières du pays d'accueil, des frontières et des distances car il a le sentiment de s'inscrire dans cet événement exceptionnel. Les acteurs de ces camps se disent changés à jamais par cette expérience.

Le phénomène des camps olympiques de la jeunesse exprime ainsi une situation de complexité. Le fonctionnement non linéaire des Jeux, à cause de son caractère quadriennal et sportif, se confronte au souhait des camps olympiques de la jeunesse d'une collaboration soutenue entre l'événement sportif et la région d'accueil, à partir de son patrimoine culturel et historique. Les Jeux ne sont plus seulement un outil au service du sport de compétition, mais un processus de refonte sur les rapports entre le touriste et les lieux touristiques de la ville olympique. Cette approche touristique et sportive permet la construction de l'identité des camps olympiques de la jeunesse et notamment, ses rapports avec la société afin de repenser les fondements de la 


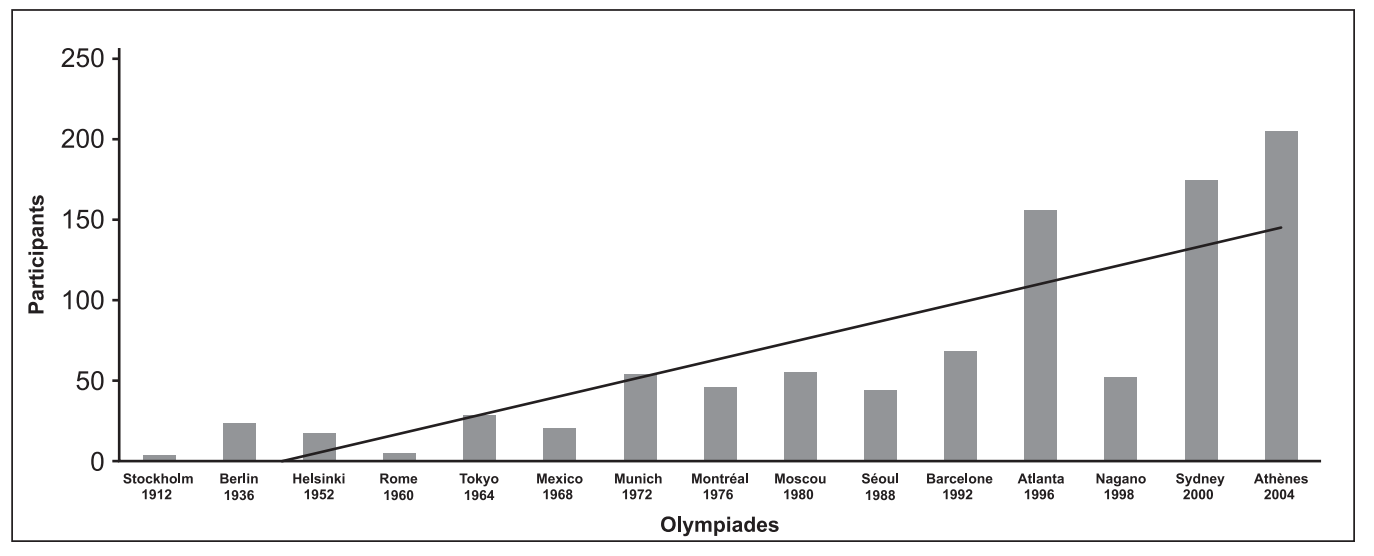

ILLUSTRATION 2 : Nombre de Comités nationaux olympiques au camp de la jeunesse depuis 1912 (source : compilation des auteurs). connaissance olympique. Le fait nouveau observé est le tourisme. Celui-ci stimule un intérêt de plus en plus grand en ce qui concerne l'identité olympique, car «le tourisme est une migration de personnes libres » (Boyer, 1999: 13).

\section{Discussion}

Cet aboutissement positif du tourisme dans le développement de l'événement olympique par l'intermédiaire des camps olympiques de la jeunesse se heurte néanmoins à une image réductrice et négative qui continue encore de dévaloriser le tourisme olympique, le touriste et le spectateur : l'impact des Jeux sur l'environnement. Toutefois, cela ne doit pas limiter la construction d'une politique d'ouverture olympique au tourisme, qui consiste à définir ou à réaliser, avec le soutien des camps olympiques de la jeunesse, un «projet de connaissance » et non un « objet de connaissance» (Le Moigne, 2004). En ce sens, trois étapes importantes se dégagent.

De 1952 à la fin des années 1960, l'accent est mis sur une sensibilisation des jeunes à l'olympisme comme culture commune. Cette approche militante matérialisée par la table ronde à la suite des $\mathrm{JO}$ de Londres en 1948 affirme un souci de former "l'esprit olympique» par une approche communautaire de la culture. Elle est concrétisée tacitement en 1968 par une "convention culturelle olympique» dont les objectifs principaux sont la sauvegarde de la culture olympique par la diversité des représentants et le développement de la compréhension mutuelle entre les peuples composant le Mouvement olympique. L'éducation et la culture deviennent les garants de la promotion de l'olympisme et d'une coopération culturelle multiforme. Il s'agit également de préserver et d'exploiter, en le valorisant, un patrimoine culturel national dans la perspective de la richesse de la solidarité entre CNO présents (illustration 2). Les camps de la jeunesse servent d'atouts à une campagne de marketing territorial, que nous observons dès 1912 sur un plan historique, mais qui se renforce avec la mise en scène de l'identité de la ville dès 1968 avec Mexico et son patrimoine historique et culturel. Le point d'aboutissement de cette phase d'exploitation de l'image olympique est l'autonomie de l'organisation qui mise sur une coopération culturelle entre olympisme et patrimoine par la jeunesse nationale et internationale des camps.
Les années 1970 sont des années de consolidation des actions engagées. Elles constituent surtout des années de réflexion sur le concept «d'identité du camp olympique de la jeunesse». Constatant que la construction olympique engagée par les membres ne revêt aucune dimension culturelle, les organisateurs des Jeux entreprennent une campagne de sensibilisation. En 1972, un «programme-cadre» est créé par une politique culturelle basée sur le passé comme construction de l'avenir où l'olympisme devient le socle. Par exemple, la vasque olympique est allumée à Montréal en 1976 par la jeunesse canadienne en signe d'unité nationale entre un francophone et une anglophone. L'olympisme vient panser l'héritage national et permet la création d'une identité culturelle olympique dont les camps se font les promoteurs. Par ailleurs, les camps se dotent de symboles identitaires : valorisation de la Journée olympique (23 juin) avec la promotion des sports par la jeunesse depuis 1948, drapeau du camp de la jeunesse avec une forte dominante du symbole olympique et un quotidien pour le camp, etc. Il est à noter que le caractère promotionnel développe ici une approche plus éducative que culturelle grâce à l'olympisme. Cette situation résume très bien l'ambition de la démarche de la ville organisatrice et du CIO, qui font des camps de la jeunesse des ambassadeurs au sein d'une stratégie de marketing territorial à partir des valeurs olympiques et du patrimoine national (architecture, culture et coutumes). Il s'agit de mobiliser la jeunesse nationale et internationale comme acteur de promotion du territoire exposé ou d'effectuer des stratégies marketing liées au territoire. Cette jeunesse y contribuera ainsi de l'amont (le diagnostic) à l'aval (la mise en ouvre opérationnelle de politiques d'animation de réseaux d'ambassadeurs).

Depuis les années 1980, les camps sont les promoteurs d'une identité culturelle olympique en construction. Il est intéressant de souligner la multiplication d'actions en faveur de la culture olympique, du patrimoine, de l'éducation, et de la jeunesse. Toutes prennent un aspect pragmatique comme fondement d'un principe nouveau, car accueillir les JO doit être une formidable opportunité pour promouvoir la richesse des territoires exposés grâce au marketing territorial. L'identité culturelle olympique est moins un héritage qu'un avenir commun. S'il n'y a pas de projet de charte culturelle, les organisateurs se contentent d'une déclaration sur les objectifs 
culturels et réaffirment la prise de conscience et la mise en valeur de l'identité culturelle olympique. Aux Jeux de Séoul en 1988, les participants souhaitent aller plus loin et impliquer le CIO. Ils rédigent en fin de camp une déclaration qui porte sur l'avenir du camp olympique de la jeunesse : «nous réclamons donc que le CIO, en tant qu'autorité responsable, ajouta à la Charte olympique, une clause obligeant les organisateurs des JO d'été et d'hiver d'ouvrir un camp international de jeunes » (SLOOC, 1989 : 474).

Le processus de la jeunesse aux Jeux décrit ci-dessus prend une tournure particulièrement riche. Du 29 août au 3 septembre 1994 se tient à Paris le Congrès olympique de «l'Unité», à l'issue duquel le Mouvement olympique fait de l'environnement un nouveau pilier de la philosophie de l'olympisme. Ce dernier est inscrit dans la Charte olympique à partir de $1996 \mathrm{au}$ même titre que le sport et la culture. Enfin, lors de la Session olympique d'Istanbul en 1999, l'environnement est considéré comme un enjeu d'avenir (Nidegger, 2002 : 511-512). En 2000, les organisateurs des Jeux de Sydney affirment la nécessité de construire un "mouvement d'avenir». Leur priorité est de développer les échanges et les débats sur les problématiques de l'olympisme (Monnin, 2000 : 32-33). Réservé dans l'immédiat après-guerre, le positionnement des camps de jeunesse s'infléchit donc au début des années 2000, en même temps que l'olympisme culturel et éducatif se construit. Une nouvelle orientation de son approche culturelle se dessine : celle du multiculturalisme. Les initiatives des camps (création de divers supports de promotion, animation de conseil, etc.) sont autant d'occasions pour les responsables des camps de jeunes de rentrer en contact avec leurs homologues internationaux et d'échanger leurs impressions.

Dans ce cadre, l'ouverture vers l'olympisme, facteur d'une internationalisation progressive, s'impose comme une nécessité. L'objectif est de lister des actions prioritaires qui, si elles étaient mises en place, permettraient aux territoires de profiter des retombées directes. Cette attractivité touristique n'est autre que l'héritage olympique (Gold et Gold, 2007). Les responsables sont soutenus dans cette démarche par les hiérarchies olympiques. Les questions de la réconciliation et de la coopération mondiale, imprégnées peu ou prou d'une dimension missionnaire (Mintzberg, 1998 : 328), apparaissent comme des priorités pour les CNO. L'ouverture des camps aux problématiques internationales en général est surtout le moyen de s'ouvrir aux grandes questions du temps présent. L'olympisme ne peut se permettre de regarder le monde se construire sans y prendre part, à l'image du développement durable. Il s'agit de "participer activement et consciemment à la construction de la société » (Charte olympique, 2013 : 11). Le CIO, quant à lui, insiste sur la nécessité de trouver dans le camp olympique de la jeunesse «une vie fraternelle» perçue comme source de rapprochement et d'échange. C'est donc bien la question de la modernisation de l'olympisme qui apparaît dans la genèse et dans les enjeux des camps de la jeunesse aux JO, même si certains $\mathrm{y}$ adhèrent avec moins d'enthousiasme que d'autres, comme en témoigne, par exemple, l'hésitation avec laquelle Moscou l'intègre dans son rapport officiel. (Novikov et OCOG Moscou, $1981: 488$ )
La notion de bonheur et de bien-être inhérente à la philosophie de l'olympisme était déjà présente en 1912 et a été reprise avec un aspect convivial comme moyen universel de communication dans le but de renforcer l'image attractive des JO par le biais des camps de la jeunesse. En déployant cette stratégie de la découverte de la ville d'accueil et du bonheur, les responsables olympiques s'approprient l'ambiance des camps et la transmettent aux locaux et aux visiteurs internationaux. Plusieurs outils de communication ont été mis en place pour atteindre cet objectif tels que des campagnes publicitaires qui associent des lieux patrimoniaux de la ville aux dynamiques olympiques. Dès lors, la question de l'olympisme, qui contient la thématique plus générale de l'ouverture internationale et des relations entre les peuples, devient pour les camps de la jeunesse un enjeu éducatif et un moyen pédagogique privilégié. De nouvelles activités sont proposées aux jeunes, de nouveaux types d'échanges encouragent l'enrichissement culturel et renouvellent leur cadre de fonctionnement habituel. L'orientation de l'olympisme permet à ces camps de se diversifier et ainsi, d'asseoir une légitimité.

\section{Conclusion}

À partir de 1996, la plupart des CNO s'inscrivent dans un processus d' "olympisation des continents", tel que le qualifie Nicolas Chamerois. Ce processus s'affirme progressivement, car l'olympisme apparaît comme une réalité à construire. Les camps de la jeunesse peuvent ainsi participer activement et originalement aux nouvelles attentes sociales et sociétales. Ce sont des lieux d'activité et d'accompagnement à la connaissance et à la compréhension de l'olympisme dans un territoire au système culturel cohérent. Leur internationalisation procure un réservoir éducatif intéressant ainsi qu'un moyen de modernisation pour une organisation qui cherche à retrouver du crédit. En ce sens, l'ouverture de l'olympisme et du Mouvement olympique peut être considérée comme une "nécessité devenue vertu» sans pour autant se traduire par une surabondance spatiale et culturelle. Ce mouvement d'expansion olympique, connu sous le nom d' "olympisation», se traduit par un jeu subtil entre le donné local de la ville d'accueil et les données des valeurs de l'olympisme qui symbolise une société globale. Les camps de la jeunesse aux JO sont donc associés à l'histoire du CIO comme patrimoine olympique dont profite la ville organisatrice pour son potentiel touristique.

Par conséquent, le CIO s'octroie une orientation culturelle grâce à un héritage olympique affirmé, mais ce quil'oblige à coopérer avec d'autres organisations internationales (Commission européenne et UNESCO) afin d'internationaliser son action sur les plans médiatique, culturel et éducatif. En effet, le camp de la jeunesse semble subir une érosion dans son nombre de participants. En 1912, 1200 jeunes y participent officiellement, tandis qu'en 1952, à Helsinki, un maximum est atteint avec 2191 participants. Cependant, depuis quelques décennies, le nombre de participants décroît de manière inquiétante. C'est à Sydney qu'il s'avère le plus bas avec seulement 381 participants. À Athènes (2004), ce chiffre remonte, un peu, pour atteindre 450 participants grâce notamment au travail fourni par la Commission européenne. Sans ce rapprochement, le nombre de jeunes aurait sans doute été équivalent à celui de Sydney. 


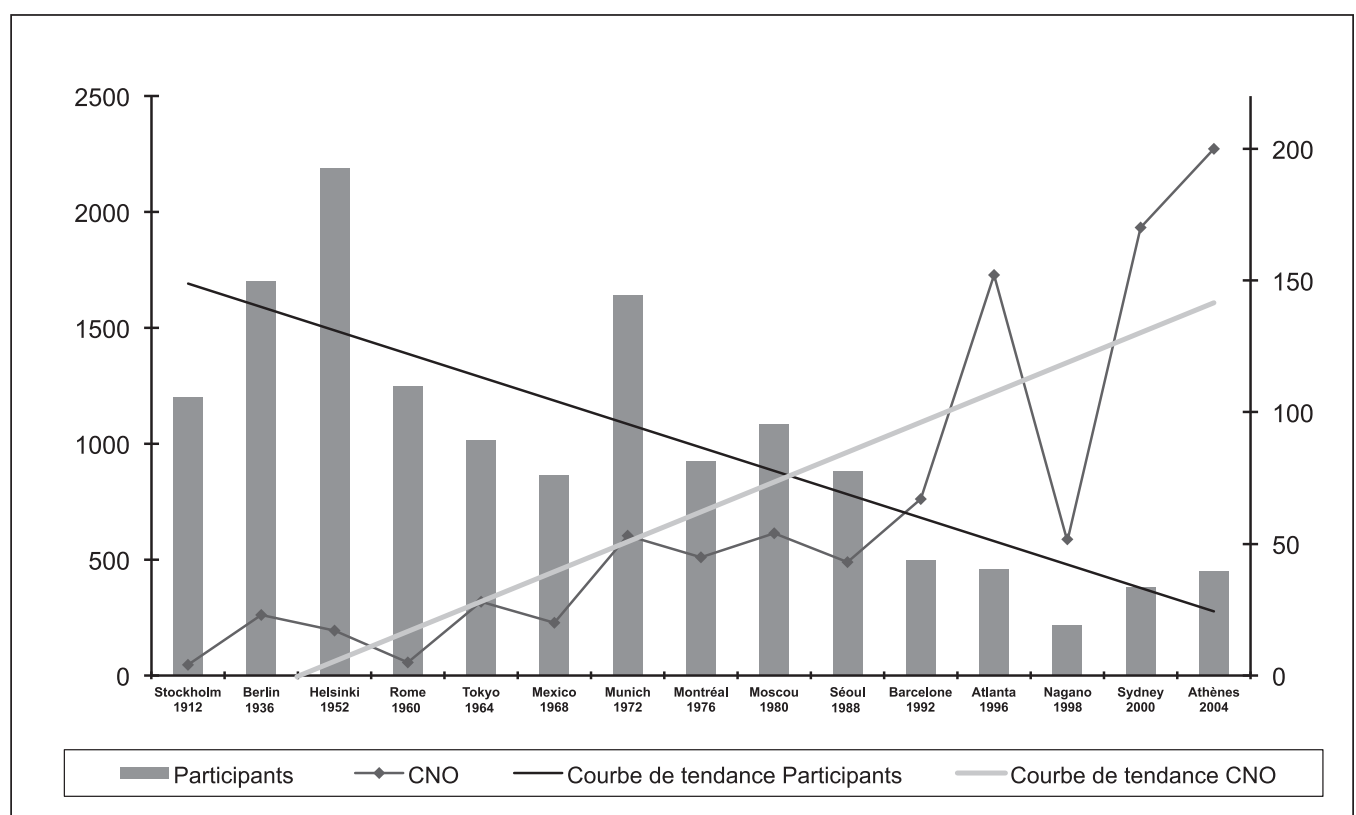

ILLUSTRATION 3 : Participants et Comités nationaux olympiques présents au camp de la jeunesse depuis 1912

(source : compilation des auteurs).
Concernant le nombre de CNO, il apparaît que les nations participantes sont de plus en plus représentées. Athènes (2004) enregistre la plus forte représentation avec deux cents participants. Si l'on tente de comparer ce phénomène avec la baisse du nombre des participants, on s'aperçoit qu'ils sont complètement à l'opposé l'un de l'autre. Les CNO ont fait le choix aujourd'hui d'envoyer uniquement le nombre de représentants demandé, soit deux, ce qui leur assure une prise en charge des jeunes envoyés par le COJO, au détriment du nombre de jeunes touchés par le message olympique (illustration 3).

La retranscription des valeurs olympiques par le biais des Jeux dans le but de valoriser une culture nationale et territoriale représente un coût financier de plus en plus lourd pour la ville organisatrice ainsi que pour les CNO participants. Par conséquent, les camps de la jeunesse se recentrent sur les valeurs et principes olympiques au détriment du nombre de participants. En revanche, dans une stratégie d'externalisation de l'image de la ville organisatrice, l'héritage ou la patrimonialisation sportive et culturelle devient un slogan de marketing auprès des populations nationales et internationales qui a été auparavant testé sur les camps de la jeunesse. Ainsi, l'emblème des Jeux de Sydney en 2000 représente «la silhouette d'un athlète aux formes et aux couleurs typiquement australiennes. Les boomerangs et la suggestion du soleil et des roches, associés aux couleurs de la baie de Sydney, des plages et de la terre rouge de l'intérieur du pays, évoquent les paysages uniques d'Australie et ses habitants d'origine. L'éclair qui transforme la silhouette de l'Opéra de Sydney en un jet de fumée s'échappant d'une torche olympique rappelle l'emblème de la candidature de Sydney» (CIO, 2000). Cet emblème destiné à des fins touristiques n'associe plus la jeunesse, mais le monde aux Jeux, conformément au choix des CNO de diminuer le nombre de participants aux camps de la jeunesse.
Les camps de la jeunesse ont construit un objet spécifique, au sein duquel les notions les plus marquantes de l'olympisme, sa culture et ses activités sont orientées depuis les années 1960 vers une attractivité du territoire local. En effet, la découverte de ce patrimoine développe un tourisme sportif et culturel dont les valeurs de convivialité et de bonheur sont soutenues par l'échange et le partage au niveau national et international. Acteurs de processus sociaux successifs, ces camps démontrent un savoir-faire, une pratique saine de l'olympisme sur un territoire donné qui leur octroie un statut spécifique dans le Mouvement olympique comme repère spatial et temporel lors de chaque Jeux.

\section{Références}

AUGUSTIN, Jean-Pierre et Jacques ION (1993) Des loisirs et des jeunes: cent ans de groupements éducatifs et sportifs, Paris : Éditions ouvrières, $145 \mathrm{p}$.

BADEN-POWELL, Robert (1920) À l'école de la vie, Paris : Neuchâtel, Delachaux et Niestlé, $3^{\mathrm{e}}$ édition.

BERGVALL, Erik (1912) The Official Report of the olympic Games of Stockholm 1912 - the Fifth Olympiad, Organising Committee for the Olympic Games in Stcokholm in 1912 Sveriges Olympiska Kommitté, Stockholm: Wahlstrom \& Widstrand. 1117 p. «ttp://doc.rero.ch/ record/32184, consulté le 29 janvier 2015.

BOYER, Marc (1999) Le tourisme de l'an 2000, Lyon : Presses universitaires de Lyon, $265 \mathrm{p}$.

BROHM, Jean-Marie (2008) 1936, Jeux olympiques à Berlin, Bruxelles: André Versailles, $244 \mathrm{p}$.

Bulletin du Comité international olympique (1950) «Texte des regrets exprimés par la délégation allemande", Revue Olympique, n 23-24, octobre-décembre, p. 21.

CHAMEROIS, Nicolas (2002) La mondialisation des Jeux olympiques - de Séoul (1988) à Sydney (2000), thèse de doctorat, Géographie, Université de Franche-Comté, CERSO, Besançon. 
CLASTRES, Patrick (2002) «La refondation des Jeux olympiques au Congrès de Paris (1894) : initiative privée, transnationalisme sportif, diplomatie des États», Relations Internationales, $\mathrm{n}^{\circ}$ 111, p. 327-345.

CLASTRES, Patrick (2008) Jeux Olympiques : Un siècle de passions, Paris : Les Quatre Chemins, 123 p.

Charte olympique (2013) Comité International Olympique, Lausanne, 9 septembre, $110 \mathrm{p}$.

CIO - Comité international olympique (2000), «L'emblème Sydney 2000 », CIO, ‘www.olympic.org), consulté le 18 décembre 2014.

CIO - Comité international olympique (1948) Procès verbal, archives du CIO, p. 17.

CIO - Comité International Olympique (1968) «Historique J.O. Mexico, membres, protocole, camp de la jeunesse, olympia dienst, sport pour tous, etc. », Lausanne, chemise : CIO J.O.-1968 S-GENER, p. 17.

COOB'92 - Comité d'organisation des Jeux Olympiques de 1992 à Barcelone (1992) Rapport officiel des Jeux de la XXVe Olympiade Barcelone 1992, Barcelone : COOB'92, 5 vol., http://doc.rero.ch/ record/209506 ?ln=frs, consulté le 29 janvier 2015.

COJO Munich - Comité d'organisation des Jeux Olympiques de 1972 à Munich (1974) Die Spiele : le rapport officiel du Comité organisateur des Jeux de la XXe Olympiade Munich 1972, 3 vol., 〈http://doc.rero.ch/ record/209523 ?ln=frs, consulté le 29 janvier 2015.

COUBERTIN, Pierre de (1996, Rééd. 1931) Mémoires Olympiques, Paris : Éd. Revue EPS, 218 p.

DIEM, Carl (1950) «L'Italie, lien créateur entre l'antiquité et les temps modernes », Bulletin du Comité International Olympique, n 19, janvier, p. 22-23 et n ${ }^{\circ} 26$, mars, p. 17.

EDSTRÖM, Johannes Sigfrid (1946) «Discours d'ouverture de la Session olympique à Lausanne", Bulletin du Comité international olympique, $\mathrm{n}^{\circ} 1$, octobre, p. 10.

EDSTRÖM, Johannes Sigfrid (1947) «Vœux», Bulletin du Comité international olympique, novembre, $\mathrm{n}^{\circ} 7, \mathrm{p} .1$.

FSGT - Fédération sportive et gymnique du travail (1936) Les amis des sports, Manifeste contre les Jeux olympiques à Berlin FSGT, Déclaration de la FSGT.

GOLD, John Robert et Margaret GOLD (2007) Olympic Cities: City Agendas, Planning, and the World's Games, 1896-2012, Londres : Routledge, 348 p.

GYGAX, Jérôme (2002) «Raisons et prétextes au boycott américain des Jeux olympiques de Moscou 1980 », Relations Internationales, $\mathrm{n}^{\circ} 112$, p. 487-509.

HATEM, Fabrice (2007) Le marketing territorial : Principes, méthodes et pratiques, Cormelles-le-Royal : EMS, $292 \mathrm{p}$.

JENNINGS, Andrew (2000) La face cachée des Jeux olympiques, Paris : Archipel, $320 \mathrm{p}$.

LE MOIGNE, Jean-Louis (2004) Le constructivisme, tome 3, Paris : L'Harmattan, $335 \mathrm{p}$.

LE MONDE (1980) «Moscou : la décision de Washington est ressentie comme un affront", Le monde, 23 janvier 1980, p. 6.

LIPPERT, Julius (1936) «Discours de M. le Commissaire d'Etat de Berlin Dr. Lippert », Bulletin officiel du Comité international olympique, $\mathrm{n}^{\circ} 32$, septembre, p. 8.

MATARD-BONNUCCI, Marie-Anne et Pierre MILZA (sous la direction de) (2004) L'Homme nouveau dans l'Europe fasciste (1922-1945) : Entre dictature et totalitarisme, Paris : Fayard, 365 p.
MINTZBERG, Henry (1998) Structure et dynamique des organisations, Paris : Les Éditions d'Organisation, $440 \mathrm{p}$.

MONNIN, Éric (2000) «Le camp international de jeunesse», Revue Olympique, $\mathrm{n}^{\circ}$ XXVII-35, octobre novembre, p. 32-33.

MONNIN, Éric (2013) De Chamonix à Sotchi. Un siècle d'olympisme en hiver, Gap : Désiris, p. 145-153.

MONNIN, Éric (2012) «The Olympic Movement's strategy for the integration of the concept of Olympic education into the education system: the French example», Educational review, vol. 64, n 3, p. 333-351.

MONNIN, Éric (2012) «International Olympic Comittee», dans John NAURIGHT et Charles PARRISH (sous la direction de), Sports around the World: History, Culture, and Practice, Santa Barbara : ABC-Clio, p. 23-26.

NIDEGGER, Jean-Blaise (2002) «Olympisme, environnement et relations internationales ", Relations Internationales, $\mathrm{n}^{\circ} 112$, hiver.

NOVIKOV, I.T et OCOG Moscou - Organising Committee for the Olympic Games in Moscow in 1980 (1981) Games of the XXII Olympiad : Official Report of the Organising Committee of the Games of the XXII Olympiad, Moscow, 1980, Moscow: Fizkultura i Sport. 3 vol., ‘http://doc.rero.ch/record/209482 ?ln=fr`, consulté le 29 janvier 2015.

OCOG Montréal - Organising Committee for the Olympic Games in Montreal in 1976 (1978) Games of the XXI Olympiad Montreal 1976 : Official Report - Ottawa: COJO 76, cop. 3 vol., http://doc.rero.ch/ record/209527? ln=fr , consulté le 29 janvier 2015.

OCOG Mexico - Organising Committee for the Olympic Games in Mexico in 1968 (1969) Mexico 68 : Official Report, Mexico : Comité organisateur des Jeux de la XIXe Olympiade, 5 vol., 〈http://doc.rero.ch/ record/209515 ?ln=fr», consulté le 29 janvier 2015.

PEPPARD, Victor et James RIORDAN (1993) Playing Politics : Soviet Sport Diplomacy to 1922, London : Jai Press Inc., 184 p.

POLYCARPE, Cyril (2013) «Le Comité international olympique, entre contraintes et enthousiasme (1939-1952)», dans Luc ROBENE (sous la direction de) Le sport et la guerre XIXe et XXe siècle, Rennes : Presses universitaires de Rennes, p. 455-463.

Revue olympique (1998) NAGANO 1998 - La Gastronomie aux Jeux olympiques. Les CNO à Cancun, Lausanne : Comité international olympique, p. 30.

RICHETER, Friedrich et OCOG Berlin - Organising Committee for the Olympic Games in Berlin in 1936 (1937) Official Report of the Games of the XI Olympiad in Berlin in 1936, Berlin: W. Limpert, cop. 1223 pages, 〈http://doc.rero.ch/record/32362 ?ln=fr〉, consulté le 29 janvier 2015.

RIOUX, Jean-Pierre et Jean-François SIRINELLI (sous la direction de) (1997) Pour une histoire culturelle, Paris : Seuil, $455 \mathrm{p}$.

SLOOC - Comité d'organisation des Jeux Olympiques de 1988 à Séoul (1989) Jeux de la XXIV ème Olympiade Séoul 1988 rapport officiel, Séoul : Comité d’organisation des Jeux olympiques de Séoul, 2 vol., 〈http://doc.rero.ch/record/209533 ?ln=fr〉, consulté le 29 janvier 2015.

SOBRY, Claude (1993) «Les grandes manifestations et installations sportives prétextes à l'aménagement du territoire ", Hommes et terres $d u$ Nord, tome 2, 1993, p. 69-73. 